\title{
Phase-field model of Hele-Shaw flows in the high-viscosity contrast regime
}

\author{
A. Hernández-Machado, ${ }^{1}$ A. M. Lacasta, ${ }^{2}$ E. Mayoral,${ }^{3}$ and E. Corvera Poiré ${ }^{3}$ \\ ${ }^{1}$ Departament ECM, Facultat de Física, Universitat de Barcelona, Diagonal 647, E-08028 Barcelona, Spain \\ ${ }^{2}$ Departament de Física Aplicada, Universitat Politècnica de Catalunya, Avinguda Dr. Marañon 44, E-08028 Barcelona, Spain \\ ${ }^{3}$ Departamento de Física y Química Teórica, Facultad de Química, UNAM Ciudad Universitaria, \\ México, Distrito Federal 04510, Mexico
}

(Received 4 February 2003; published 29 October 2003)

\begin{abstract}
A one-sided phase-field model is proposed to study the dynamics of unstable interfaces of Hele-Shaw flows in the high viscosity contrast regime. The corresponding macroscopic equations are obtained by means of an asymptotic expansion from the phase-field model. Numerical integrations of the phase-field model in a rectangular Hele-Shaw cell reproduce finger competition with the final evolution to a steady-state finger.
\end{abstract}

DOI: 10.1103/PhysRevE.68.046310

PACS number(s): 47.54.+r, 05.10.-a, 47.55.Mh

\section{INTRODUCTION}

The characterization of the dynamics of morphologically unstable interfaces is one of major problems of nonequilibrium phenomenology [1]. Some relevant examples of interfaces that grow out of equilibrium are dendritic growth, directional solidification, flow in porous media, electrodeposition, bacterial colony growth, and two-fluid flow in a Hele-Shaw cell. The latter example is also called the Saffman-Taylor problem and has played a central role in this field, both because of its relative simplicity and because of its potential importance in oil recovery. It has been widely studied both experimentally and theoretically.

Even if the Saffman-Taylor problem is mathematically simple in relation to other problems, it has a moving boundary condition which makes it a free-boundary problem. The corresponding equations have been solved analytically for very short times by means of a linear stability analysis and for the steady-state finger shape by means of conformal mapping techniques $[2,3]$. Some analytical results have also been obtained for the dynamics of intermediate time [4]. Numerically there are several techniques, most of them involving integral boundary methods [5-7]

The so-called phase-field models have been introduced within the context of solidification to study the dynamics from the linear regime to the long time behavior [8]. These models are based on the introduction of a mesoscopic equation for an order parameter (the phase-field). This equation is coupled to other physical fields (such as a thermal field). The advantage of this method is that one does not have to explicitly trace the interface. It is a field model for all values of the order parameter that varies continuously from one phase to the other. One has to identify the locus of points with a given value of the order parameter, which is arbitrarily chosen to be the interface. The use of a mesoscopic model, for which the interface has a small width $\epsilon$, is justified as long as in the sharp interface limit $\epsilon \rightarrow 0$ the correct macroscopic equations are recovered. Recently, the concept of phase-field models has been used in a broader sense to include any model which contains continuous fields that are introduced to describe phases separated by diffuse interfaces. Phase-field models have been used in a wide range of problems such as viscous fingering, roughening, vesicles, pinch-off and reconnection in a Hele-Shaw cell and intracellular dynamics [9-14].

In general, the phase-field models have been considered for symmetric situations where the characteristic parameters (such as the thermal diffusivity) are identical in both phases. This gives rise to the so-called two-sided symmetric models. Very recently, Karma [15] has proposed a phase-field model of the one-sided type (with zero diffusion in one phase) to simulate quantitatively microstructural pattern formation of alloy solidification. For the viscous fingering problem with arbitrary viscosity contrast, a phase-field model has been introduced in Ref. [9]. Such a phase-field model, which is a two-sided model, is useful to describe the problem of viscous fingering except in the high-viscosity contrast regime. This regime is experimentally relevant since typically the pushing fluid is either air or other fluid of negligible viscosity. For such a regime, a proper model was lacking and this is what we are presenting in this paper; a one-sided phase-field model for the high-viscosity contrast regime of the viscous fingering problem.

Our model contains an equation for an order parameter. It is model $B$ of Ginzburg-Landau phenomenology [16]. Instead of the coupling of the order parameter to a physical field through a second equation, we include a boundary condition such that the interface becomes unstable. This is done by means of a ramp that creates a flux from the boundary. To consider a one-sided model, we only need to neglect changes in the order parameter in one of the two phases. The model could also be relevant for dendritic growth at very small undercooling by introducing anisotropy $[17,18]$.

Our phase-field model has the advantage of being very simple to implement on a computer and contains a complete description of all the nonlinear and nonlocal properties of the macroscopic model. We show how the macroscopic equations of the problem are obtained from the phase-field model in the sharp interface limit. This is done by means of the matched asymptotic expansion method. We then present numerical solutions showing how our phase-field model reproduces the main features of the viscous fingering problem such as the dynamic competition of modes and the formation of a steady-state finger. This makes the model an attractive tool to use to study problems that would not be easily feasible with traditional methods such as the propagation of viscous fingering in the presence of quenched disorder. 


\section{THE MODEL}

\section{The viscous fingering problem}

In the Saffman-Taylor problem both fluids are governed by Darcy's law, which relates the fluid velocity to the pressure gradient [2]. When the low-viscosity fluid displaces the high-viscosity fluid, the interface between both fluids is unstable. When the pushing fluid is considered to have zero viscosity, Darcy's law states that the pressure on the pushing fluid is constant and all that remains to be solved are the equations for the viscous fluid subject to the proper boundary conditions at the fluid-fluid interface. This is called the highviscosity contrast regime and in this regime the equations for the displaced viscous fluid are

$$
\begin{gathered}
\nabla^{2} p=0, \\
v_{n}=-K \nabla p \cdot \hat{n}, \\
\Delta p=\gamma \kappa .
\end{gathered}
$$

Equation (1) is the Laplace equation in the bulk, where $p$ is the pressure of the viscous fluid. At the interface, there are two boundary conditions: the continuity equation, Eq. (2) and the Gibbs-Thomson condition, Eq. (3). $v_{n}$ is the velocity normal to the interface. $K$ is the permeability of the viscous fluid, $K=b^{2} / 12 \mu$ where $b$ is the separation between the plates, and $\mu$ is the viscosity of the fluid that is being pushed. $\Delta p$ is the pressure of the viscous fluid minus the constant pressure at the zero viscosity fluid, which without loss of generality can be taken equal to zero. $\kappa$ is the local curvature at the interface and $\gamma$ is the surface tension. These three equations also describe solidification in the quasistatic limit of small undercooling by introducing anisotropy. In what follows, we present the equations for our phase-field model and show how it reproduces the above equations in the sharp interface limit.

\section{Phase-field model}

Our phase-field model contains a time-dependent Ginzburg-Landau equation for a conserved order parameter and includes a boundary condition that makes the interface unstable. The equation reads

$$
\frac{\partial \phi}{\partial t}=\boldsymbol{\nabla} \cdot\left[M_{0} \boldsymbol{\nabla}\left(-\phi+\phi^{3}-\epsilon^{2} \nabla^{2} \phi\right)\right] .
$$

The local order parameter $\phi$ adopts the equilibrium values $\phi_{e q}=1$ (air phase) and $\phi_{e q}=-1$ (viscous fluid phase). At the interface, $\phi$ varies continuously from one phase to the other. The parameter $M_{0}$ has a constant value in each phase and is zero in air,

$$
M_{0}= \begin{cases}M & \text { if } \phi<0 \\ m=0 & \text { if } \phi \geqslant 0 .\end{cases}
$$

The air phase can be pulled toward the viscous fluid. An unstable interface is developed by maintaining a slope in the order parameter close to the interface, as the case shown in

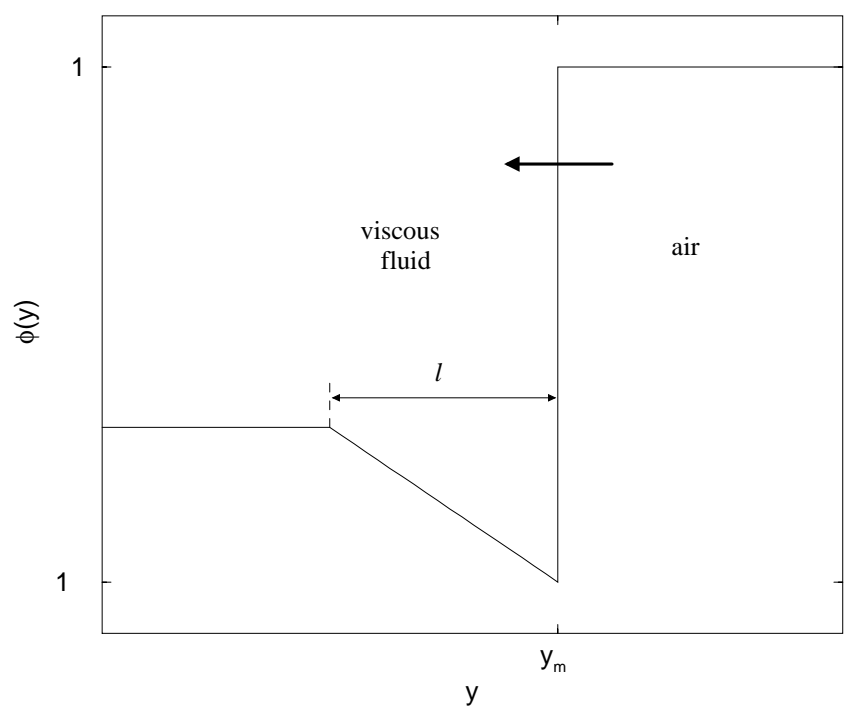

FIG. 1. Scheme of the initial profile prepared with a ramp that will be maintained during the temporal evolution.

Fig. 1. This situation can be created by initially preparing the system with a profile of the form

$$
\phi(x, y)= \begin{cases}1 & \text { if } y>y_{m}, \\ -1-\alpha\left(y-y_{m}\right) & \text { if } y_{m}-l<y \leqslant y_{m}, \\ -1+\alpha l & \text { if } y \leqslant y_{m}-l,\end{cases}
$$

and fixing the value $\phi_{f}=-1+\alpha l$ behind the interface up to a distance $l$ throughout all the temporal evolution. This slope represents the driving force of the system. The parameter $\alpha$ controls the slope and the finger's growth velocity. For convenience we will refer to the air as the plus phase and to the viscous fluid as the minus phase.

\section{THE SHARP INTERFACE LIMIT}

In this section we obtain the macroscopic equations for the viscous fingering problem in the high-viscosity contrast regime by means of an asymptotic expansion of the phasefield model in the sharp interface limit $\epsilon \rightarrow 0[19,20]$.

Our starting point is Eq. (4), used in the study of a conserved order parameter $\phi$. The chemical potential is given by

$$
\mu(\phi)=\mu_{B}-\epsilon^{2} \nabla^{2} \phi=-\phi+\phi^{3}-\epsilon^{2} \nabla^{2} \phi .
$$

We divide the space into an outer and an inner region. We assume that $\phi= \pm \phi_{e q}+O(\epsilon)$ far from the interface. $\epsilon$ is considered to be a small parameter and we expand all the variables $a(\mathbf{r}, t)$ around the value $\epsilon=0$ in the outer region. We obtain

$$
a(\mathbf{r}, t)=a_{0}+\epsilon a_{1}+\epsilon^{2} a_{2}+\cdots .
$$

For the interfacial region or inner region, we adapt our coordinate system using time-dependent curvilinear coordi- 
nates. The interfacial points are given by the curvilinear coordinates $u$, which is the normal distance to the interface, and $s$, which is the arclength. Because the natural dimension in the inner region must be small, we introduce the variable $w$ defined as $w=u / \epsilon$. Thus, in the sharp interface limit, when $\epsilon \rightarrow 0$, the inner region goes from $w \rightarrow-\infty$ to $w \rightarrow+\infty$. We use the corresponding inner fields $A(w, s, t)$ in the inner region and the corresponding expansion is

$$
A(w, s, t)=A_{0}+\epsilon A_{1}+\epsilon^{2} A_{2}+\cdots .
$$

When we take the limit of the sharp interface, $\epsilon \rightarrow 0$, the conditions for the fields $a$ and $A$, from the expansions to $i$ th order in $\epsilon$ are

$$
\begin{gathered}
\lim _{w \rightarrow-\infty} A_{i}=\lim _{u \rightarrow-0} a_{i}, \\
\lim _{w \rightarrow-\infty} \partial_{w} A_{i+1}=\lim _{u \rightarrow-0} \partial_{u} a_{i} .
\end{gathered}
$$

Due to the fact that $m=0$ in air, the matching condition is only imposed in the viscous phase.

In the inner region, we introduce the order parameter $\widetilde{\phi}$ such that $\widetilde{\phi}(u(t), s, t)=\phi(\mathbf{r}, t)$, therefore

$$
\partial_{t} \phi=\partial_{t} \widetilde{\phi}+\partial_{t} u \partial_{u} \widetilde{\phi}
$$

We rescale time as $\tau=\epsilon t$ since we work in the quasistatic approximation, where the characteristic times for interface motion are much larger than the characteristic times for the diffusion to take place. The local curvature $\kappa=-\nabla^{2} u$ is positive when a bump of the $\phi>0$ phase protrudes into the $\phi<0$ phase. Starting from Eq. (12), using the Laplacian operator in curvilinear coordinates $\nabla^{2}=\partial_{u}^{2}-\kappa \partial_{u}+\partial_{s}^{2}$, and making the corresponding variable changes, we have

$$
\epsilon \partial_{\tau} \phi-\frac{v}{\epsilon} \partial_{w} \phi=M\left[\frac{1}{\epsilon^{2}} \partial_{w}^{2} \mu(\phi)-\frac{\kappa}{\epsilon} \partial_{w} \mu(\phi)+\partial_{s}^{2} \mu(\phi)\right],
$$

where we have dropped the tildes. The normal velocity $v=$ $-\partial_{t} u$ is positive if the phase with a negative order parameter goes into the phase with a positive order parameter. This variable is also expanded in powers of $\epsilon$.

For the chemical potential $\mu$, the inner expansion in terms of $\phi$ (to order $\epsilon^{2}$ ) is given by

$$
\mu(\phi)=\mu_{0}+\epsilon \mu_{1}+\epsilon^{2} \mu_{2},
$$

with

$$
\begin{gathered}
\mu_{0}=\mu_{B_{0}}-\partial_{w}^{2} \phi_{0}, \\
\mu_{1}=\mu_{B_{0}}^{\prime} \phi_{1}-\partial_{w}^{2} \phi_{1}+\kappa \partial_{w} \phi_{0}, \\
\mu_{2}=\frac{1}{2} \mu_{B_{0}}^{\prime \prime}\left(\phi_{1}\right)^{2}+\mu_{B_{0}}^{\prime} \phi_{2}-\partial_{w}^{2} \phi_{2}+\kappa \partial_{w} \phi_{1}-\partial_{s}^{2} \phi_{0},
\end{gathered}
$$

where $\mu_{B_{0}}=\mu_{B}\left(\phi_{0}\right)$. The prime represents the derivative of $\phi$ evaluated at $\phi_{0}$.
For the region far from the interface (outer region), the length scale involved is much greater than $\epsilon$, so we can use a common time-independent coordinate system. In the viscous fluid region the dynamical equation for the order parameter is simply

$$
\epsilon \partial_{\tau} \phi=M \nabla^{2} \mu(\phi),
$$

where $\mu(\phi)$ is given by Eq. (7).

\section{Inner region}

We now proceed to solve the equations for the inner region, Eqs. (13)-(15). We also use the matching conditions, Eqs. (10) and (11). Solutions that obey $\phi(0)=0$ and $\partial_{w} \phi_{0}(-\infty)=0$ are required.

Order $\epsilon^{-2}$. For the inner region, the dynamical equation to lowest order in $\epsilon,\left(\epsilon^{-2}\right)$ is taken from Eq. (13),

$$
\partial_{w}^{2} \mu_{0}=0 .
$$

Here we have taken into account the expansion for $\mu$. The previous expression has a solution $\mu_{0}=m_{0}+n_{0} w$. The requirement that $\mu_{0}$ must be finite for $w \rightarrow-\infty$ implies that $n_{0}=0$. Finally, we consider $m_{0}=0$ and then $\phi_{e q}= \pm 1$. Therefore, $\mu_{0}=0$ in the inner region.

Order $\epsilon^{-1}$. Taking the first-order terms $\epsilon^{-1}$ from the dynamical equation in the inner region, Eq. (13), we have

$$
-v_{0} \partial_{w} \phi_{0}=M \partial_{w}^{2} \mu_{1},
$$

since $\mu_{0}=0$. Integrating Eq. (18) in $w$ we find

$$
-v_{0} \phi_{0}=M \partial_{w} \mu_{1}+n_{1} .
$$

By evaluating between the limits $w=-\infty$ and $w=\infty$, Eq. (19), we obtain

$$
-2 \phi_{e q} v_{0}=M \partial_{w} \mu_{1}(-\infty),
$$

where $2 \phi_{e q}$ is the order parameter change between the two phases and we have only the contribution of the viscous fluid phase on the right-hand side. Using Eq. (11) we have $\partial_{w} \mu_{1}(-\infty)=\partial_{u} \mu_{0}(-0)=0$. From Eq. (20), $v_{0}$ also vanishes and Eq. (19) gives $n_{1}=0$.

By integrating Eq. (19) in $w$, we find that $\mu_{1}=\mu_{1}(-\infty)$ is a constant. In order to obtain $\mu_{1}(-\infty)$, we use its expression from Eq. (15) and we multiply both sides of this expression by $\partial_{w} \phi_{0}$ and integrate in $w$

$$
\begin{aligned}
\mu_{1}(-\infty) \int d w \partial_{w} \phi_{0}= & \int d w \partial_{w} \phi_{0}\left(\mu_{B_{0}}^{\prime}-\partial_{w}^{2}\right) \phi_{1} \\
& +\kappa \int d w\left(\partial_{w} \phi_{0}\right)^{2}
\end{aligned}
$$

The function $\partial_{w} \phi_{0}$ is known as the Goldstone mode and is related to the translational invariance of the interface. The equation for $\phi_{0}$, written as a function of the rescaled variable $w$, is $\mu_{B_{0}}-\partial_{w}^{2} \phi_{0}=0$. Differentiating with respect to $w$ we obtain an equation for $\partial_{w} \phi_{0}$, which is $\left(\mu_{B_{0}}^{\prime}-\partial_{w}^{2}\right) \partial_{w} \phi_{0}=0$. 
So the Goldstone mode is a zero eigenvector of the linear operator $\mu_{B_{0}}^{\prime}-\partial_{w}^{2}$. By doing integration by parts, the first term on the right-hand side of Eq. (21) vanishes and we obtain

$$
\mu_{1}(-0)=\frac{\gamma}{\phi_{e q}} \kappa
$$

where $\gamma=\frac{1}{2} \int d w\left(\partial_{w} \phi_{0}\right)^{2}$ is the surface tension and we have used the matching condition for $\mu_{1}(-0)$ from Eq. (10). Taking into account the fact that at the interface $p(-0)$ $=\phi_{e q} \mu_{1}(-0)$ we obtain Eq. (3).

Order $\epsilon^{0}$. In order to obtain the continuity equation, we need to go to the next order. The dynamical equation to order $\epsilon^{0}$ in the inner region is

$$
-v_{1} \partial_{w} \phi_{0}=M\left(\partial_{w}^{2} \mu_{2}-\kappa \partial_{w} \mu_{1}+\partial_{s}^{2} \mu_{0}\right) .
$$

Integrating Eq. (23) in the direction normal to $w$ we find

$$
-2 \phi_{e q} v_{1}=M \partial_{w} \mu_{2}(-\infty)=M \partial_{u} \mu_{1}(-0),
$$

where we have used the matching condition, Eq. (10), and the fact that $\partial_{w} \mu_{1}(-\infty)=0$ and $\mu_{0}=0$. Equation (24) could be written as Eq. (2) in terms of the pressure at the interface of the viscous fluid, where $K=M /\left(2 \phi_{e q}^{2}\right)$.

\section{Outer region}

Order $\epsilon^{-2}$. The dynamical equation in the outer region to the lowest order $\epsilon^{-2}$ is

$$
\nabla^{2} \mu_{0}=0 .
$$

The boundary condition far from the interface is then $\mu_{0}$ $=0$. We previously found that $\mu_{0}=0$ for the inner solution at the interface. The only solution satisfying both conditions is $\mu_{0}=0$. It follows that $\phi_{0}=\phi_{e q}$ in the plus phase and $\phi_{0}$ $=-\phi_{e q}$ in the minus phase. This was to be expected since the lowest order in the expansion corresponds to the solution of the flat interface.

$\operatorname{Order} \epsilon^{-1}$. The dynamical equation for the order $\epsilon^{-1}$ is

$$
\nabla^{2} \mu_{1}=0 .
$$

At order $\epsilon$ the order parameter and the chemical potential are proportional and from Eq. (26) we obtain Eq. (1).

\section{NUMERICAL RESULTS}

We have numerically integrated Eqs. (4) and (5) with $\epsilon$ $=1$ and $M=1$ on a rectangular lattice of vertical size $L_{y}$ $=200$ and mesh size $\Delta x=1$, with periodic boundary conditions in the $x$ direction and reflecting boundary conditions in the $y$ direction. The system has been prepared with a horizontal interface containing some perturbations in order to be destabilized. The profile in the vertical direction is formed by Eq. (6) with $l=10$. As was mentioned before, during the evolution we maintain a slope by fixing the value $\phi_{f}=-1$ $+\alpha l$ behind the interface up to a distance $l$ measured from the tip of the most advanced finger. (a)
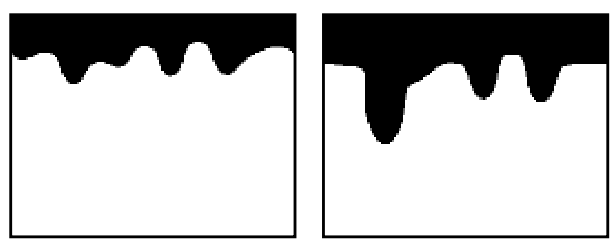

(c)

(b)
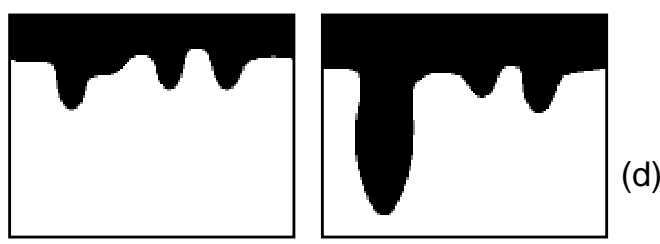

FIG. 2. Finger development and competition for $\alpha=0.04$ corresponding to early times: $t=100$ (a), 500 (b), 1000 (c), and 2000 (d).

\section{Finger competition}

First, we are interested in the generation and subsequent competition of fingers during the early stages of the evolution. A wide system of size $L_{x}=128$ has been considered and we have prepared an initial corrugated interface formed by the superposition of several modes of random amplitude. In Fig. 2 we show a typical evolution. It is seen that fingers develop from the random initial configuration. Some modes grow, some modes decay, and finger competition begins. Both features have been observed in theoretical and experimental studies of the viscous fingering problem. The competition process continues until only one of the fingers survives.

In order to better visualize the competition process we have prepared a second initial condition consisting of two well-formed fingers, in which one of them is a bit larger than the other. In Fig. 3 we observe how the largest finger grows at the expense of the other, which moves backwards, becoming smaller, and eventually disappearing.

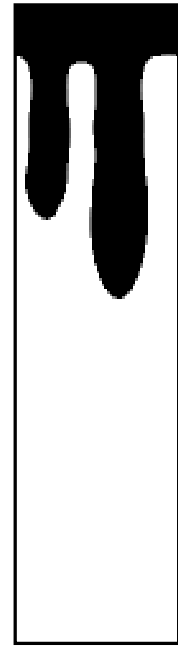

(a)

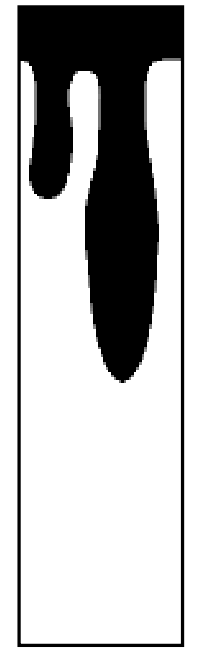

(b)

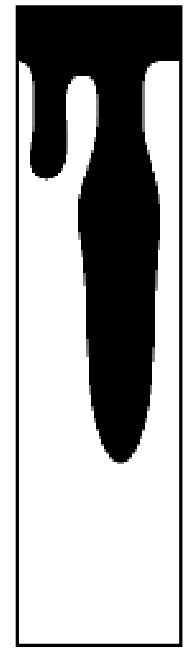

(c)

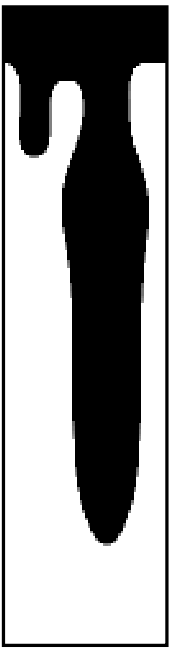

(d)
FIG. 3. Finger competition process for two initially well-formed fingers with $\alpha=0.04$ and system width $L_{x}=64$. The patterns are separated by time intervals of 1000 . 
(a)

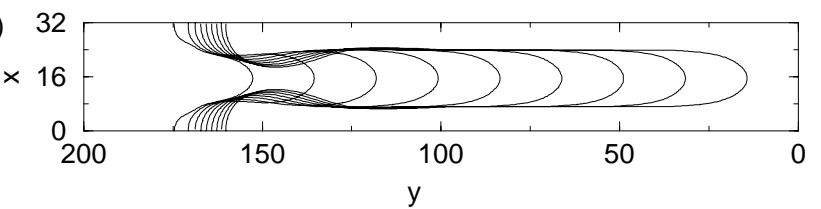

(b)

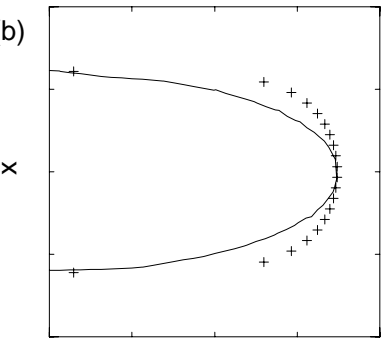

y (c)

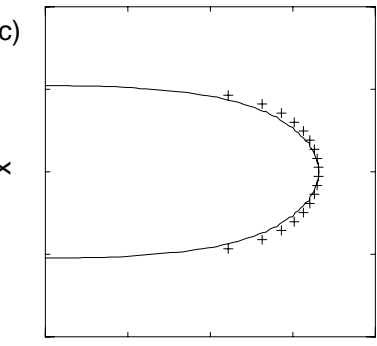

y
FIG. 4. (a) Evolution of a single finger in a channel, plotted at time intervals of 750, for $\alpha=0.035$. (b) and (c) Numerical results (lines) and Saffman-Taylor solution (symbols) are presented for two values of $\alpha$ that lead to two different finger widths (b) $\lambda=0.61$ and (c) $\lambda=0.53$.

\section{Steady-state finger}

The width of the steady-state finger is expected to go to one-half of the channel width as the velocity of the finger tip increases [3,21]. To better explore this situation, we have considered a narrow channel of width $L_{x}=32$, prepared with an initial condition that gives a single finger. We have analyzed the temporal evolution of the finger for different tip velocities corresponding to different values of the parameter $\alpha$. In agreement with known results, higher velocities led to narrower fingers. An example of the interface evolution is shown in Fig. 4(a). In Figs. 4(b), (c) we compare the finger shapes obtained numerically for two different values of $\alpha$ with the theoretical shape for the Saffman-Taylor finger [2]

$$
y=y_{t i p}-\frac{L_{x}(1-\lambda)}{2 \pi} \ln \left[\frac{1}{2}\left(1+\cos \frac{\pi\left(2 x-L_{x}\right)}{\lambda L_{x}}\right)\right] \text {, }
$$

$\lambda$ being the ratio of the width of the finger to the width of the channel. To determine $\lambda$ from our numerical results, we have evaluated the average width of the finger throughout the evolution, in a strip of thickness $e=4$ placed at a distance 40 from the tip. For high enough tip velocities, our numerical results are in agreement with the Saffman-Taylor solution since they correspond to values of $\lambda$ close to $1 / 2$ [Fig. 4(c)], where surface tension effects are negligible. Also, the expected deviation from the Saffman-Taylor solution is observed for wider fingers in qualitative agreement with Ref. [3].

We have measured the finger-width and the finger-tip velocity $v$ for different values of the parameter $\alpha$. The results, shown in Fig. 5, are in qualitative agreement with experimental results of Pitts [21] and Saffman-Taylor [2] and with the numerical results of McLean and Saffman [3]. We ob-

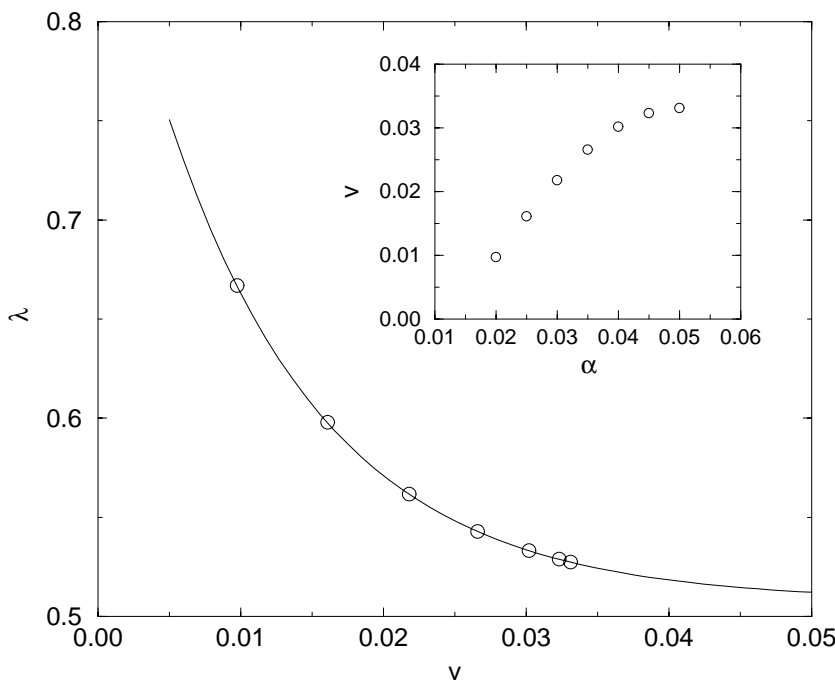

FIG. 5. Finger-width $\lambda$ vs velocity $v$. Solid line is a guide to the eye. Inset shows the dependence of $v$ on the parameter $\alpha$.

serve that $\lambda$ tends to one-half of the channel width as the velocity increases [22].

\section{CONCLUSIONS}

A one-sided phase-field model to describe the dynamic evolution of unstable interfaces for Hele-Shaw flows in the high-viscosity contrast regime has been proposed. The mesoscopic model contains a field equation for a conserved order parameter (model $B$ of Ginzburg-Landau phenomenology) and a boundary condition that drives the interface out of equilibrium. An asymptotic expansion to derive the macroscopic equations has been performed. The phase-field model has been numerically integrated and we have analyzed different stages of the dynamics. We observe how from a random perturbation to the interface, fingers develop. Modes grow and compete dynamically and the competition ends in a single steady-state finger. The width of this finger goes to one-half of the channel width as the velocity increases. This is in agreement with experiments and the existent theory. We have verified that the shape of the finger tip is in good agreement with the parametric solution of Saffman and Taylor when the finger width is close to one-half of the channel width. Also for larger width the shape is in qualitative agreement with the fingers found by Mc Lean and Saffman. We believe that our model could be a useful tool to study situations that cannot be easily tackled with traditional methods, like integro-differential equations, such as the effect introduced by quenched disorder.

\section{ACKNOWLEDGMENTS}

A.H.M. and A.M.L. acknowledge financial support from the Dirección General de Enseñanza Superior (Spain) under Project Nos. BFM2000-0628-C03-01 and BFM2000-0624C03-02, respectively. E.C.P. acknowledges financial support from PAPIIT through Grant No. IN117802-02. 
[1] Branching in Nature, edited by V. Fleury, J.-F Gouyet, and M. Léonetti (Springer-Verlag, Berlin, 2001); E. Ben-Jacob and H. Levine, Adv. Phys. 49, 395 (2000); J.P. Gollub and J.S. Langer, Rev. Mod. Phys. 71, S396 (1999); Solids Far From Equilibrium, edited by C. Godrèche (Cambridge University Press, Cambridge, 1992); P. Pelcé, Dynamics of Curved Fronts (Academic, New York, 1988).

[2] P.G. Saffman and G.I. Taylor, Proc. R. Soc. London, Ser. A 245, 312 (1958); D. Bensimon, L. Kadanoff, S. Liang, B.I. Shraiman, and C. Tang, Rev. Mod. Phys. 58, 977 (1986).

[3] J.W. McLean and P.G. Saffman, J. Fluid Mech. 102, 455 (1981).

[4] M. Siegel and S. Tanveer, Phys. Rev. Lett. 76, 419 (1996).

[5] G. Tryggvason and H. Aref, J. Fluid Mech. 136, 1 (1983); 154, 287 (1985).

[6] D. Jasnow and J. Vinals, Phys. Rev. A 40, 3864 (1989); 41, 6910 (1990); J. Casademunt, D. Jasnow, and A. HernándezMachado, Int. J. Mod. Phys. B 6, 1647 (1992); E. Pauné, M. Siegel, and J. Casademunt, Phys. Rev. E 66, 046205 (2002).

[7] T.Y. Hou, J.S. Lowengrub, and M.J. Shelley, J. Comput. Phys. 114, 312 (1994).

[8] J.S. Langer, in Directions in Condensed Matter Physics, edited by G. Grinstein and G. Mazenko (World Scientific, Singapore, 1986), p. 165; R. Kobayashi, Physica D 63, 410 (1993); G. McFadden, A. Wheeler, R. Braun, S. Coriell, and R. Sekerka, Phys. Rev. E 48, 2016 (1993); K.R. Elder, M. Grant, N. Provatas, and J.M. Kosterlitz, ibid. 64, 021604 (2001).

[9] R. Folch, J. Casademunt, A. Hernández-Machado, and L. Ramírez-Piscina, Phys. Rev. E 60, 1724 (1999); 60, 1734 (1999).
[10] R. Gonzalez-Cinca, R. Folch, R. Benitez, L. Ramirez-Piscina, J. Casademunt, and A. Hernandez-Machado, e-print cond-mat/0305058; Advances in Condensed Matter and Statistical Mechanics, edited by E. Korucheva and R. Cuerno (Nova Science, New York, in press).

[11] A. Hernández-Machado, J. Soriano, A.M. Lacasta, M.A. Rodríguez, L. Ramírez-Piscina, and J. Ortín, Europhys. Lett. 55, 194 (2001).

[12] T. Biben and C. Misbah, Phys. Rev. E 67, 031908 (2003).

[13] H.G. Lee, J.S. Lowengrub, and J. Goodman, Phys. Fluids 14, 492 (2002); 14, 514 (2002).

[14] J. Kockelkoren, H. Levine, and W.J. Rappel, e-print cond-mat/0305577.

[15] A. Karma, Phys. Rev. Lett. 87, 115701 (2001).

[16] P.C. Hohenberg and B.I. Halperin, Rev. Mod. Phys. 49, 435 (1977).

[17] R. Almgren, W.-S. Dai, and V. Hakim, Phys. Rev. Lett. 71, 3461 (1993).

[18] J.L. Mozos and H. Guo, Europhys. Lett. 32, 61 (1995).

[19] An asymptotic expansion was presented in Ref. [20] within the context of driven diffusive systems. In this case the destabilization of the interface has a different origin related to the presence of an external field and a two-sided symmetric model is considered.

[20] C. Yeung, J.L. Mozos, A. Hernández-Machado, and D. Jasnow, J. Stat. Phys. 70, 1149 (1993).

[21] E. Pitts, J. Fluid Mech. 97, 53 (1980).

[22] In our model we have not included the film effect observed by P. Tabeling, P.G. Zocchi, and A. Libchaber, J. Fluid Mech. 177, 67 (1987). 